\title{
The Effect of Lower Stratospheric Shear on Baroclinic Instability
}

\author{
Matthew A. H. Wittman and Andrew J. Charlton \\ Department of Applied Physics and Applied Mathematics, Columbia University, New York, New York \\ LORENZO M. POLVANI \\ Department of Applied Physics and Applied Mathematics, and Department of Earth and Environmental Sciences, \\ Columbia University, New York, New York
}

(Manuscript received 14 October 2005, in final form 5 May 2006)

\begin{abstract}
Using a hierarchy of models, and observations, the effect of vertical shear in the lower stratosphere on baroclinic instability in the tropospheric midlatitude jet is examined. It is found that increasing stratospheric shear increases the phase speed of growing baroclinic waves, increases the growth rate of modes with low synoptic wavenumbers, and decreases the growth rate of modes with higher wavenumbers. The meridional structure of the linear modes, and their acceleration of the zonal mean jet, changes with increasing stratospheric shear, but in a way that apparently contradicts the observed stratosphere-troposphere northern annular mode (NAM) connection. This contradiction is resolved at finite amplitude. In nonlinear life cycle experiments it is found that increasing stratospheric shear, without changing the jet structure in the troposphere, produces a transition from anticyclonic (LC1) to cyclonic (LC2) behavior at wavenumber 7. All life cycles with wavenumbers lower than 7 are LC1, and all with wavenumber greater than 7 are LC2. For the LC1 life cycles, the effect of increasing stratospheric shear is to increase the poleward displacement of the zonal mean jet by the eddies, which is consistent with the observed stratosphere-troposphere NAM connection. Finally, it is found that the connection between high stratospheric shear and high-tropospheric NAM is present by NCEP-NCAR reanalysis data.
\end{abstract}

\section{Introduction}

Observations of downward-propagating anomalies in the annular mode index (Baldwin and Dunkerton 1999) have spurred much recent research into the dynamical link between the stratosphere and the troposphere. Recent studies (Plumb and Semeniuk 2003; Christiansen 2003; S. Hardiman 2005, personal communication) have investigated the propagation of the anomalies from high in the stratosphere down to the tropopause, but have not accounted for the communication of annular mode index anomalies from the tropopause to the ground, which seems to occur by a fundamentally different process. Referring to the height-time picture of the downward-propagating NAM index (Baldwin and Dunkerton 2001, their Fig. 1), we note that whereas the descent of anomalies to the tropopause follow a broad

Corresponding author address: L. M. Polvani, 500 W. 120th Street, Room 216, Columbia University, New York, NY 10027. E-mail:1mp@columbia.edu slanted path, indicating a slow descent of long-lived anomalies, the communication of anomalies from the tropopause to the underlying troposphere appears to occur on a much faster time scale, with sporadic, shortlived anomalies appearing throughout the troposphere while the tropopause anomaly persists. That the response of the troposphere is mediated by baroclinic eddies is suggested by several facts: the time scale of a few days over which tropospheric anomalies occur following the descent of a stratospheric anomaly to the tropopause is consistent with the time scale of baroclinic instability and the tropospheric response is concentrated near the ground and the tropopause, consistent with the structure of Eady-like baroclinic modes. Recent work strengthens the suggestion that baroclinic instability is sensitive to the state of the stratosphere: Polvani and Kushner (2002) found a response in the tropospheric annular mode to different stratospheric polar vortex strengths in long integrations with a dynamical core, and that the response was mediated by synoptic eddies (Kushner and Polvani 2004); Charlton

DOI: $10.1175 / J A S 3828.1$

(C) 2007 American Meteorological Society 

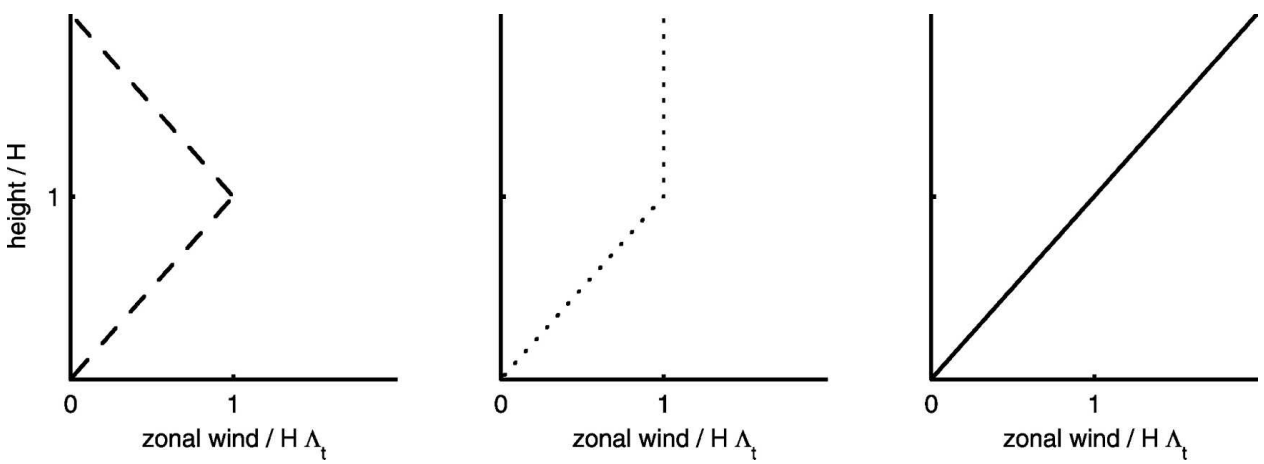

FIG. 1. The vertical profile of zonal wind specified as the basic state for the 1D modified Eady problem. Negative, zero, and positive stratospheric shears are denoted by the dashed, dotted, and solid lines, respectively.

et al. (2004) documents synoptic-scale tropospheric responses to different stratospheric conditions in models, and Limpasuvan et al. (2004) documents synoptic-scale responses to stratospheric sudden warmings in observations; Limpasuvan and Hartmann (2000) found that synoptic eddies were an important driver of the tropospheric annular mode index; and Wittman et al. (2004, hereafter W04) shows surface annular mode responses to changing polar vortices in life cycle experiments. This paper focuses specifically on the role of vertical shear in the lower stratosphere, since, as we will show, changes to the vertical shear at the top boundary have a simple effect on the linear growth of baroclinic eddies, which may determine the nonlinear development of baroclinic eddies and thus the tropospheric mean flow.

To investigate the role of baroclinic instability in communicating stratospheric anomalies to the surface, we return to the simplest model of baroclinic instability, the Eady problem. Despite its simplicity, the Eady problem constitutes a remarkably powerful description of baroclinic instability, and is well suited to investigating the effect of tropopause flow anomalies because the instability relies on the resonance between counterpropagating Rossby waves at the ground and the tropopause (Heifetz et al. 2004), changes to conditions at the tropopause will result in changes to the properties of the upper wave, and thus whole growing mode. The Eady problem has also been shown to apply in cases where growth is nonmodal (Rivest and Davis 1992).

We start with a modified Eady problem, in which we find the effect of increasing stratospheric shear is to increase the phase speed of the growing baroclinic modes, and to increase the growth rate of the lower synoptic wavenumbers. Because the Eady problem supplies no information about the meridional effect of the instability on the mean flow, which we need to determine if we wish to understand the origin of the annular mode signal, we turn next to the linear problem in primitive equations on the sphere. Here we find that the effect of stratospheric shear on the growth rates and phase speeds is qualitatively the same as in the onedimensional model, but that the structure of the normal modes, and the acceleration of the mean flow, do not change in a way that is consistent with the observed annular mode signal. Thus, in the next section, we consider the finite amplitude effect of baroclinic instability in life cycle experiments. Here we find that increasing stratospheric shear leads to enhanced acceleration of the jet on the poleward side, consistent with an increased annular mode response. We also find that with an initial perturbation of intermediate wavenumber, it is possible to change the life cycle from an LC1 to an LC2 by increasing the stratospheric shear. The transition from LC1- to LC2-type behavior raises new issues in understanding the dynamics underlying the transition, since we are able to induce the transition without changing the structure of the baroclinic jet in the troposphere. Finally, we examine reanalysis data and find evidence that the relationship between stratospheric shear and the annular mode response suggested by the life cycle experiments obtains.

\section{Review of the 1D problem}

A comprehensive mathematical development of the 1D quasigeostrophic (QG) Eady problem with a stratosphere is given in Müller (1991) and Blumen (1979), although their work predated the discovery of the downward propagation of annular mode index anomalies from the stratosphere. Since the Eady problem is well known, here we give only an overview of the key results; Müller (1991) gives a fuller treatment.

We consider two atmospheric layers, in which both the static stability and wind shear are constant (Fig. 1). 
TABLE 1. Relation between nondimensional and dimensional variables for the Eady problem in section 2 . Note that the variables with asterisks are nondimensional.

\begin{tabular}{lcc}
\hline \multicolumn{1}{c}{ Quantity } & $\begin{array}{c}\text { Dimensional } \\
\text { variable }\end{array}$ & $\begin{array}{c}\text { Equals factor } \times \\
\text { nondimensional } \\
\text { variable }\end{array}$ \\
\hline Height & $z$ & $H z^{*}$ \\
Horizontal displacement & $x$ & $\frac{N_{t} H}{f_{0}} x^{*}$ \\
Time & $t$ & $\frac{N_{t}}{f_{0} \Lambda_{t}} t^{*}$ \\
Stratospheric shear & $\Lambda_{s}$ & $\frac{\Lambda_{s}}{\Lambda_{t}} \lambda^{*}$ \\
Stratospheric Brunt-Väisälä & $N_{s}$ & $\frac{N_{s}}{N_{t}} n^{*}$ \\
frequency & &
\end{tabular}

The troposphere is bounded below, at $z=0$, and shares a free interface, at $z=H$, with the stratosphere above. The stratosphere is unbounded above, and the BruntVäisälä frequency of the stratosphere is fixed at its typical value of 4 times that of the troposphere $\left(N_{s}^{2}=4 N_{t}^{2}\right)$. The stratospheric shear $\Lambda_{s}$ is varied from -1 to +1 times the tropospheric shear $\Lambda_{t}$.

We nondimensionalize with the height of the tropopause $H$, the tropospheric shear $\Lambda_{t}$, the tropospheric static stability $N_{t}$, and the Coriolis parameter $f_{0}$. The relations between the nondimensional and dimensional variables are shown in Table 1; the variables shown with asterisks are nondimensional.

The nondimensional streamfunction $\Psi^{*}$ then obeys Laplacian equations

$$
\begin{gathered}
\left\{\begin{array}{c}
1 \\
n^{* 2}
\end{array}\right\}\left(\Psi_{x^{*} x^{*}}^{*}+\Psi_{y^{*} y^{*}}^{*}\right)+\Psi_{z^{*} z^{*}}^{*}=0 \\
\text { for }\left\{\begin{array}{c}
0 \leq z^{*} \leq 1 \\
z^{*} \geq 1
\end{array}\right.
\end{gathered}
$$

and boundary conditions

$$
\begin{gathered}
\Psi^{*} \rightarrow \text { const. for } z^{*} \rightarrow \infty, \\
\left.\Psi^{*}\right|_{z^{*}=1^{-}}=\left.\Psi *\right|_{z^{*}=1^{+}}, \\
\left.n^{* 2}\left(\Psi_{z^{*} t^{*}}^{*}+\Psi_{z^{*} x^{*}}^{*}-\Psi_{x^{*}}^{*}\right)\right|_{z^{*}=1^{-}} \\
=\left.\left(\Psi_{z^{*} t^{*}}^{*}+\Psi_{z^{*} x^{*}}^{*}-\lambda^{*} \Psi_{x^{*}}^{*}\right)\right|_{z^{*}=1^{+}}, \\
\Psi_{z^{*} t^{*}}^{*}-\Psi_{x^{*}}^{*}=0 .
\end{gathered}
$$

These conditions represent the disappearance of wind velocity and temperature perturbations at the top of the domain, continuity of pressure, and vertical wind fields
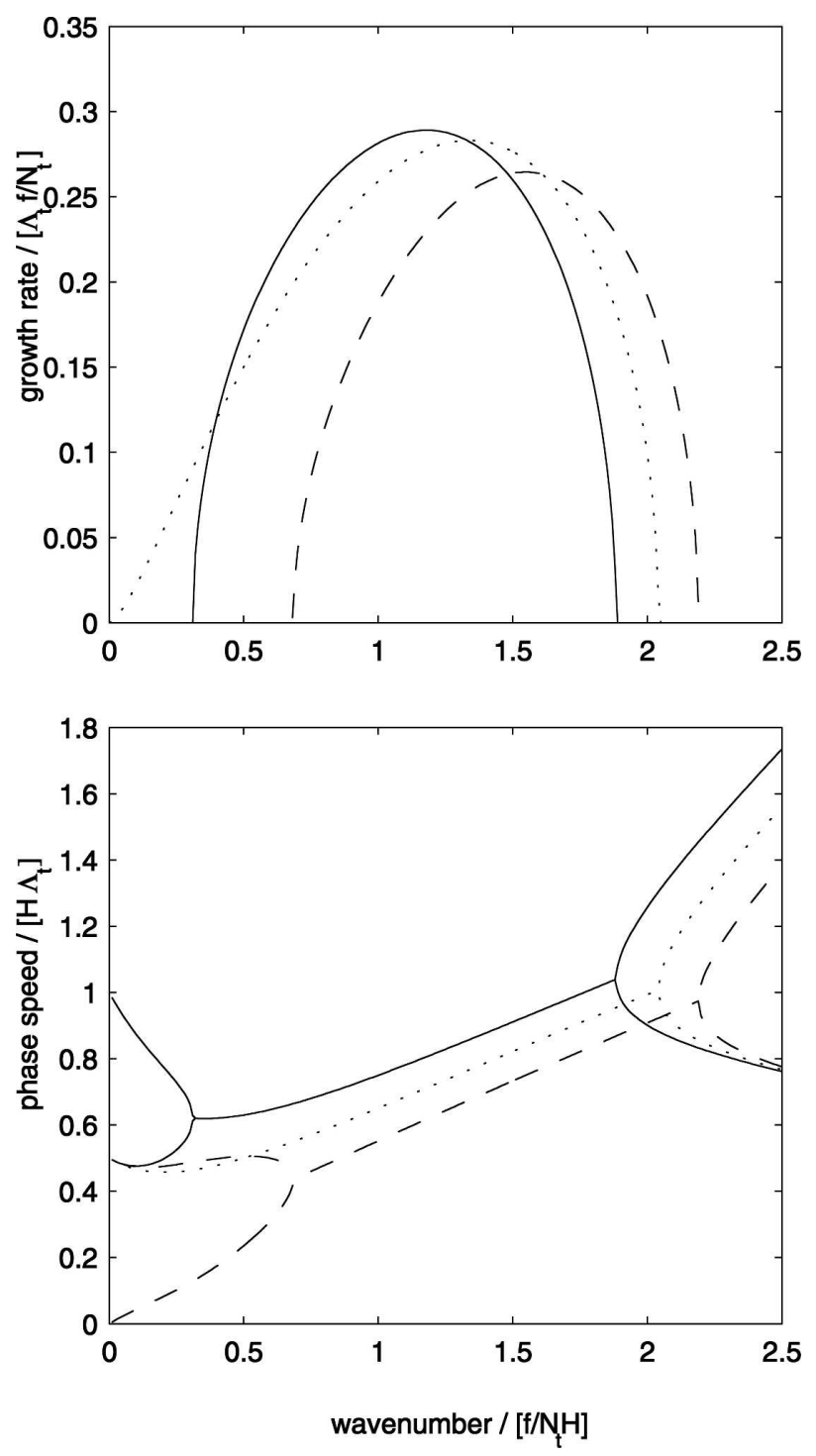

FIG. 2. Growth rate and phase speed as a function of wavenumber for the 1D modified Eady problem. Dashed, dotted, and solid lines represent negative, zero, and positive stratospheric shear, respectively.

at the interface (at $z^{*}=1$ ), and temperature conservation at the surface, respectively.

Normal mode solutions of (1) for $\lambda^{*}=-1,0,1$ (i.e., stratospheric shear equal to $-1,0$, and 1 times tropospheric shear) result in the phase speeds and growth rates shown in Fig. 2. Positive stratospheric shear shifts the growth rate curve upward and toward low wavenumbers, so that the peak wavenumber is reduced, and the growth rates of wavenumbers below the peak are increased. Negative stratospheric shear shifts the growth-rate curve downward and toward higher wavenumbers. The phase speed of the unstable waves increases monotonically with stratospheric shear at all 
unstable wavenumbers. It should be noted that extreme positive and negative stratospheric shears, beyond the range considered here, suppress growth rates at all wavenumbers (cf. Müller 1991).

The effect of increasing stratospheric shear may be understood by considering the background potential vorticity (PV) gradient at the tropopause: Bretherton (1966) gives the expression for the background QG potential vorticity gradient as

$$
q_{0 y}=-f^{2}\left(\frac{\Lambda_{s}}{N_{s}^{2}}-\frac{\Lambda_{t}}{N_{t}^{2}}\right)_{H^{-}}^{H^{+}},
$$

so that $q_{0 y}$ is positive for the range of $\Lambda_{s}$ and $N_{s}^{2}$ that we consider. Recall that $\beta$ is absent in the Eady problem. Since the phase speed of a Rossby wave is given by $c-$ $\bar{u}=-q_{0 y} / k, c-\bar{u}$ becomes less negative with increasing stratospheric shear, and a free Rossby wave at the tropopause propagates more rapidly eastward with the mean flow.

Eady baroclinic instability may be considered as the interaction between two counterpropagating free Rossby waves that propagate on the potential vorticity gradients that exist only at the lower boundary and the tropopause (Davies and Bishop 1994; Heifetz et al. 2004, hereafter H04). When stratospheric shear is increased, the phase speed of the upper wave, which is the phase speed of a free Rossby wave and is directed westward, against the mean flow, decreases. Thus the phase speed of the coupled mode, which is equal to the mean of the phase speeds of the two edge waves [H04, their Eq. (53)], increases. Hence, increased stratospheric shear leads to higher phase speeds at all wavenumbers.

When the stratospheric shear is zero, the PV gradients at each edge are equal and opposite, as in the original Eady problem. At the wavenumber at which the growth rate is a maximum (the peak wavenumber), the two phase speeds of the top and bottom waves are equal and opposite, and the phase difference between them is $\pi / 2$. The barotropic wind field associated with each edge wave is thus optimally aligned to amplify the meridional displacement of the other wave, giving the maximum growth rate. At wavenumbers less than the peak, the phase speed of each wave is higher, and without mutual interaction the two waves would tend to travel past each other. (Recall that for free Rossby edge waves, the phase speed is given by $c=-q_{0 y} / k ; c$ is positive at the bottom edge, and negative at the top edge. Additionally, at the top edge the phase speed is Doppler shifted by the nonzero wind there.) To ensure the phase locking necessary for modal growth, the phase difference between the two waves increases
(H04; Fig. 2). Correspondingly, at wavenumbers above the peak the phase speeds of the two edge waves are too low for coherence, and the phase difference must decrease, so that each wave helps the other to propagate. Away from the peak wavenumber, the growth rate decreases because some component of the wind induced by each wave helps or hinders the other's propagation, and therefore is unavailable for growing the other wave.

When stratospheric shear is increased from zero, the PV gradient at the top edge is decreased, decreasing the phase speed of the top wave. To correct for the decreased phase speed, the peak wavenumber decreases, increasing the speed of both edge waves so they cohere again. The increase in peak growth rate is due to the vertical dependence of the edge waves on wavenumber-for a free Rossby edge wave the vertical structure of the streamfunction is an exponential whose thickness is inversely proportional to wavenumber $\left[\psi(z) \propto e^{-k z}\right]$, and thus at lower wavenumbers the wind induced by one wave at the other's location increases. As pointed out in $\mathrm{H} 04$, the growth rate of a baroclinic normal mode depends both on the phase relationship between the two edge waves, which governs how effectively each wave is able to induce the other to grow, and the size of the PV gradient supporting each edge wave, which governs how much the edge wave grows as it is subjected to the influence of the other. For the values of shear considered here, the main effect on growth rate is due to the change in phase between the two edge waves. However, at higher shears the PV gradient at the top edge is reduced, reducing the growth rate.

The opposite occurs when stratospheric shear decreased below zero: the top edge PV gradient is increased so that the peak wavenumber increases to compensate for the increased edge wave speed. The maximum growth rate declines because the vertical extent of the edge waves decreases.

The cutoff that appears at low wavenumbers with nonzero stratospheric shears is due to the fact that mismatched PV gradients at the top and bottom result in mismatched phase speeds. The mismatch is worst at low wavenumbers $\left(c=-q_{0 y} / k\right)$, and the modal growth becomes impossible when the two waves can no longer compensate for the mismatch by altering their phase alignment.

The 1D QG problem provides information about the growth rate and vertical structure of the growing baroclinic wave, but yields no information about the meridional structure of the normal modes, nor the meridional transports of momentum and heat, which are crucial in determining the effect of the instability on the meridional structure of the zonal mean flow, and the effect on 

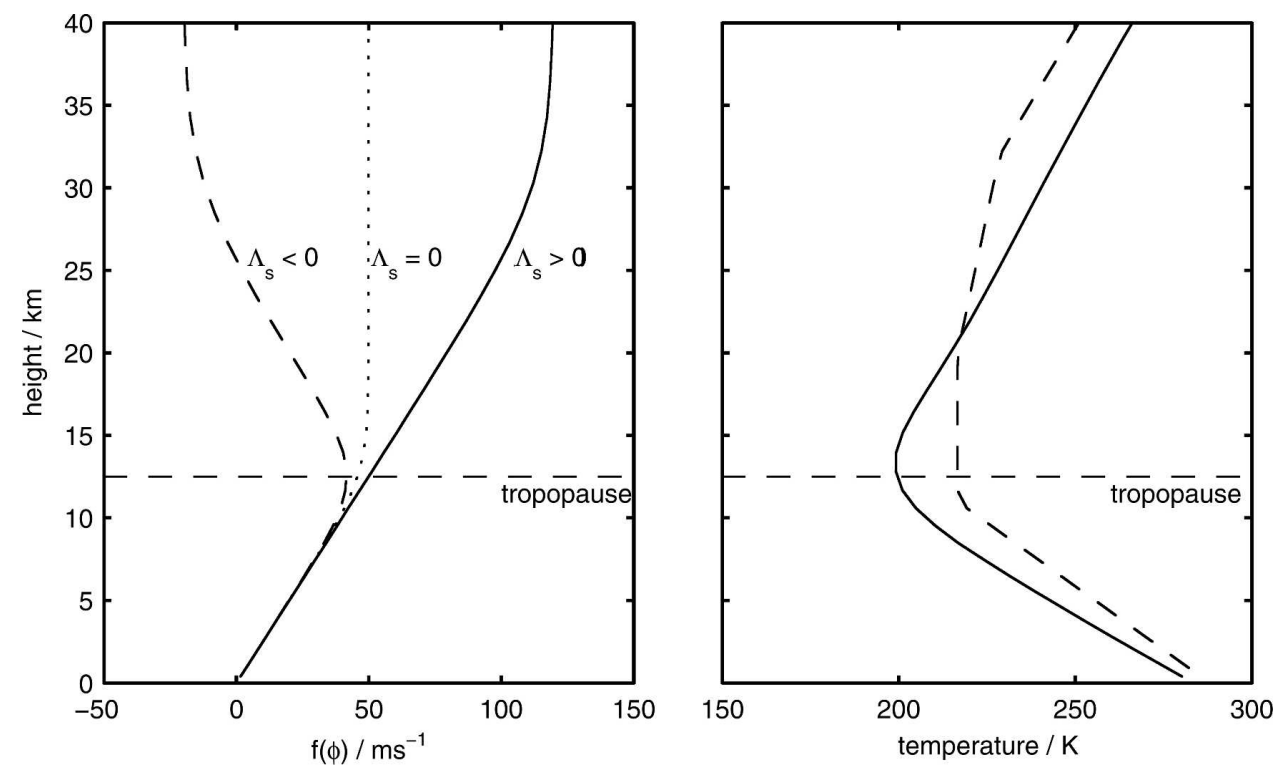

FIG. 3. Initial conditions for the linear and nonlinear primitive equations calculations. (left) The vertical profiles of the zonal wind, with dashed, dotted, and solid lines representing negative, zero, and positive stratospheric shear, respectively. (right) The solid line shows the meridional mean vertical profile specified in our initial conditions, and the dashed line represents the U.S. Standard Atmosphere, 1976, for comparison.

the annular mode index. Therefore, we turn next to a study of the analogous linear instability problem in three dimensions.

\section{Toward a meridional picture: The linear problem in primitive equations}

In the atmosphere, baroclinic instability arises in the meridionally confined midlatitude jet, and it is the momentum and heat fluxes into and out of the jet that change its structure and result in the annular mode signal-the hallmark of stratospheric influence-as the jet's latitude is changed. To understand the downward propagation of the NAM signal from the stratosphere, therefore, we must investigate the meridional structure of the growing baroclinic modes and the associated accelerations of the basic state. We therefore calculate the baroclinically unstable normal modes in the threedimensional linearized primitive equations for basic states with varying stratospheric shear.

\section{a. Basic states}

The basic-state zonal winds are specified as a function of latitude $\phi$ and log-pressure height, $z=-H$ $\log \left(p / p_{0}\right)$, where $H$ is a scale height of $7 \mathrm{~km}$. The zonal wind is given by $u(\phi ; z)=g(\phi) f(z)$, where the meridional dependence

$$
g(\phi)=\sin ^{3}\left[\pi \sin ^{2}(\phi)\right],
$$

specifies a midlatitude jet centered at $45^{\circ} \mathrm{N}$, as for the tropospheric jet in W04.

The vertical dependence $f(z)$ is specified so as to match that of the Eady problem as closely as possible (Fig. 3). Integrating the thermal wind relationship in latitude, and differentiating in height, so that

$$
\frac{\partial \theta}{\partial z}=-\frac{R}{f} \int \frac{\partial^{2} u}{\partial z^{2}} d \phi+h(z)
$$

imposes a limit on the maximum vertical curvature of the zonal wind, since $\partial \theta / \partial z$ must be positive to ensure static stability. Thus, for reasonable choices of the function of integration $h(z)$, the shear cannot change abruptly at the tropopause. To accomplish the required smooth shear profile we choose a vertical dependence of the initial zonal wind specified using the function introduced in Lindzen (1970),

$$
\begin{aligned}
f(z)= & \Lambda_{t} \frac{z}{2} \\
& +\frac{1}{2} \delta_{t}\left(\Lambda_{s}-\Lambda_{t}\right) \log \left[\cosh \left(\frac{z-z_{t}}{\delta_{t}}\right) / \cosh \left(\frac{z_{t}}{\delta_{s}}\right)\right] \\
& -\frac{1}{2} \delta_{s} \Lambda_{s} \log \left[\cosh \left(\frac{z-z_{s}}{\delta_{s}}\right) / \cosh \left(\frac{z_{s}}{\delta_{s}}\right)\right],
\end{aligned}
$$


in which $\Lambda_{t, s}$ are shears, $\delta_{t, s}$ are parameters controlling the curvature of the corners, and the $z_{t, s}$ are heights. These parameters take the values shown in Table 2. This formulation well approximates a piecewise linear function of height with three sections, as can be seen in Fig. 3. The tropospheric shear approximates that of the standard baroclinic jet of Simmons and Hoskins (1980) and Thorncroft et al. (1993, hereafter T93). The stratospheric shear $\Lambda_{s}$ is varied, as in the $1 \mathrm{D}$ problem.

The vertical profile of zonal wind has zero shear above $30 \mathrm{~km}$ in the stratosphere so that excessive zonal wind speeds do not necessitate small time steps for the numerical integration. Our results are robust to variations in this cut-off height, as well as the sharpness of the corners (within reasonable values).

The same zonal wind jet is specified in both hemispheres, and the temperature field is specified by thermal wind balance. The free function of integration $h(z)$ is chosen so that the meridional mean vertical temperature profile, denoted by $\theta(z)$, corresponds to an Eadylike arrangement of two regions of constant $N^{2}$, with a smooth transition at the tropopause:

$$
\begin{aligned}
\theta(z)= & \frac{\theta_{0}}{2}\left\{\left[1+\tanh \left(\frac{z_{t}-z}{\delta_{\theta}}\right)\right] \exp \left(\frac{N_{t}^{2} z}{g}\right)\right. \\
& \left.+\left[1+\tanh \left(\frac{z-z_{t}}{\delta_{\theta}}\right)\right] \exp \left(\frac{N_{t}^{2} z_{t}+N_{s}^{2} z}{g}\right)\right\},
\end{aligned}
$$

where the symbols are defined as before and parameter values are given in Table 2; $g$ is the standard gravitational acceleration.

\section{b. Details of the calculation}

Since a linear stability analysis of the linearized primitive equations leads to a nonseparable eigenproblem, we take inspiration from Simmons and Hoskins (1976) and numerically solve an initial value problem in a modified dynamical core whose solutions converge to the required eigenmodes and associated growth rates.

We use the "Built on Beowulf" (BOB) dynamical core described in Rivier et al. (2002). We compute with a horizontal spectral resolution of T85 and 40 vertical pressure levels, arranged to give adequate coverage of the stratosphere as in Polvani and Scott (2004); the model top is located at approximately $80 \mathrm{~km}$. For the linear normal mode calculations diffusion in time and space is set to zero. We specify initial conditions, as described above, to be a zonally symmetric function, and perturb the temperature field with a single Fourier mode of wavenumber $k=m$. The functional form of this perturbation is
TABLE 2. Parameters specifying the vertical dependence of the basic-state zonal wind and potential temperature for the calculations with the primitive equations in sections 3 and 4 .

\begin{tabular}{cc}
\hline \hline Parameter & Value(s) \\
\hline$Z_{t}$ & $12.5 \mathrm{~km}$ \\
$z_{s}$ & $30 \mathrm{~km}$ \\
$\Lambda_{t}$ & $4 \mathrm{~m} \mathrm{~s}^{-1} \mathrm{~km}^{-1}$ \\
$\Lambda_{s}$ & $-4,0,+4 \mathrm{~m} \mathrm{~s}^{-1} \mathrm{~km}^{-1}$ \\
$N_{t}^{2}$ & $1.2 \times 10^{-4} \mathrm{~s}^{-2}$ \\
$N_{s}^{2}$ & $5 \times 10^{-4} \mathrm{~s}^{-2}$ \\
$\delta_{t}$ & $3 \mathrm{~km}$ \\
$\delta_{s}$ & $5 \mathrm{~km}$ \\
$\delta_{\theta}$ & $5 \mathrm{~km}$ \\
$\theta_{0}$ & $288 \mathrm{~K}$ \\
\hline
\end{tabular}

$$
\begin{aligned}
& T^{\prime}(\phi, z) \\
& =\left\{\begin{array}{l}
\left\{\frac{1}{2}+\left[\tanh \left(\frac{\left(\phi^{\mathrm{o}}-10\right)}{5}\right)\right] /(1+\tanh 2)\right\} e^{-(z / 5)} \\
\text { for } \phi>0 \\
0 \quad \\
\text { for } \phi \leq 0
\end{array}\right.
\end{aligned}
$$

but our results are independent of the specific form of the perturbation, which governs only how fast the solution converges to the most unstable normal mode.

At every time step, each prognostic variable $X$ is Fourier transformed in the longitudinal direction:

$$
X(\lambda, \phi, p)=\sum_{k} \tilde{X}_{k}(\phi, p) e^{i k \lambda}
$$

where $(\lambda, \phi, p)$ are latitude, longitude, and pressure.

Linearization of the primitive equations requires two modifications. The zonal mean terms $(k=0)$ are held constant, and each product of two or more variables that are perturbations from the zonal mean is set to zero. We implement the first requirement by storing and resetting all of the components with $k=0, \tilde{X}_{0}$, and the second by setting the $\tilde{X}_{k \neq m}$ to zero at each time step. Thus, the only Fourier coefficients that survive are those of the initial perturbation, which evolve, and those of the zonally symmetric basic state, which remain constant.

A zonally symmetric basic state typically supports many baroclinically unstable modes at each zonal wavenumber, and the initial perturbation in general projects onto all of them. However, since each mode grows exponentially with time, the solution always converges to the most unstable mode. Since the equations are linear, we rescale all of the $k \neq 0$ coefficients by the same factor every time the perturbation amplitude exceeds 

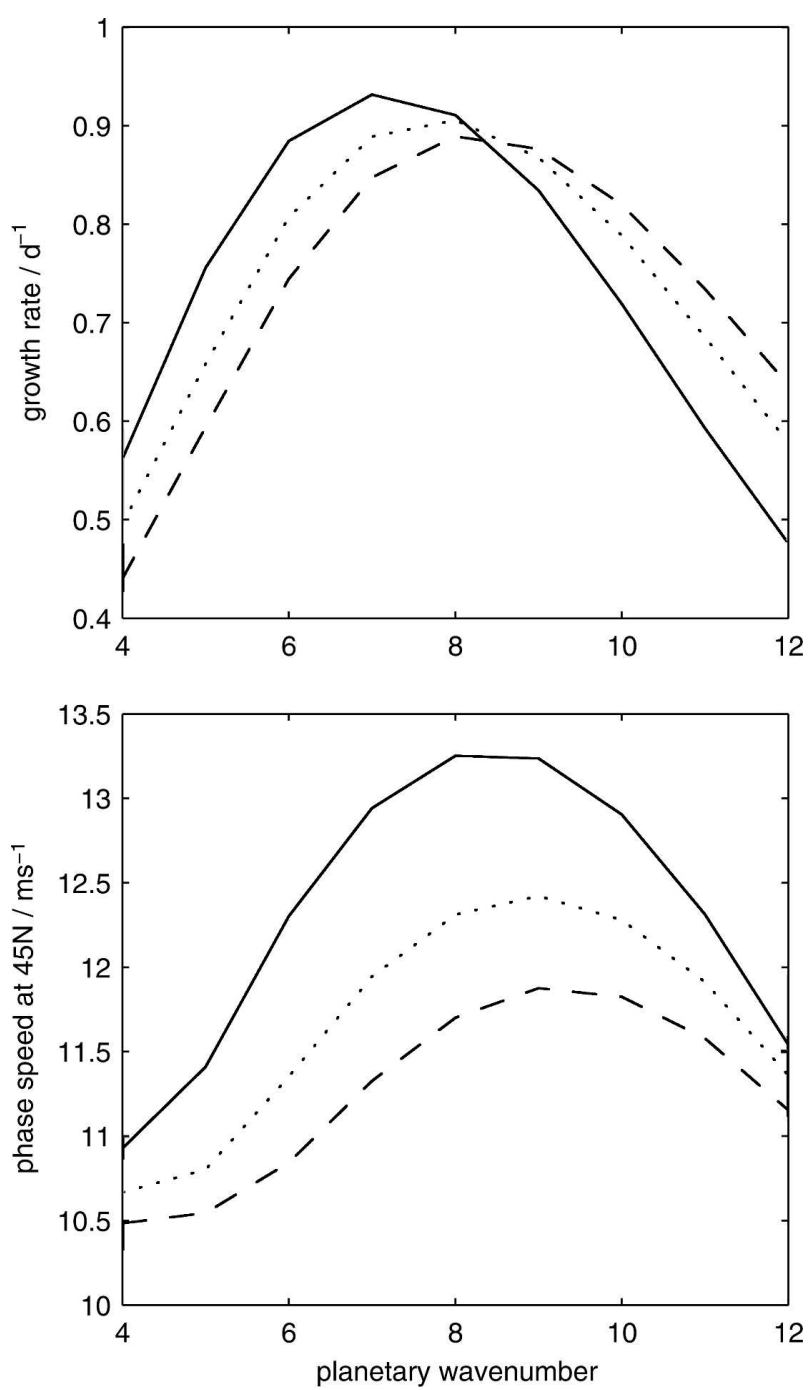

FIG. 4. Growth rate and phase speed as functions of zonal wavenumber for the linear primitive equations calculations. Dashed, dotted, and solid lines represent negative, zero, and positive stratospheric shear, respectively.

some specified threshold, so as to keep their values suitably small. Thus, we monitor the solution, which grows exponentially with periodic rescaling, until it has converged satisfactorily to the normal mode.

We then calculate the growth rate of the eddy kinetic energy (EKE) from the perturbation quantities. The EKE is calculated at each time step, and grows as the square of the perturbation quantities. The phase speed is calculated from the difference in the phase of any of the perturbation fields at successive time steps.

\section{c. Results}

From Fig. 4 we see that the qualitative features of the 1D Eady problem are preserved for a basic state with a latitudinally confined jet: the growth rate curves are shifted in the same way, and phase speed is a monotonically increasing function of stratospheric shear at all wavenumbers, although, compared with the $1 \mathrm{D}$ problem, the phase speed peaks at intermediate wavenumbers at all stratospheric shears.

Figure 5 shows the effect of increasing stratospheric shear on the most unstable mode at zonal wavenumber 7. The effect is qualitatively the same at all zonal wavenumbers, and for the range of stratospheric shears considered here, completely monotonic. Increasing stratospheric shear increases the relative amplitude of the normal mode on the poleward side of the jet, and increases the penetration of the disturbance into the stratosphere.

Figure 6 shows the corresponding accelerations, $\partial \bar{u} / \partial t$, of the basic state by the most unstable normal mode, calculated by perturbing the fully nonlinear dynamical core with the normal modes at small amplitude. The effect of increasing stratospheric shear is to increase the deceleration of the jet on its poleward flank. Increased decelerations of the basic state on its poleward side with increasing stratospheric shears correspond to the jet moving farther equatorward as the result of baroclinic instability. In observations, the highstratospheric NAM index, which corresponds to a strong polar vortex and high values of stratospheric shear (Thompson and Wallace 1998), is associated with high-tropospheric NAM index, which corresponds to a high-latitude tropospheric jet position. The accelerations of the jet by small amplitude baroclinic modes that tend to move the jet equatorward with increasing stratospheric shear appear to contradict this, implying an association between high-stratospheric NAM index and low-tropospheric NAM index.

However, this apparent contradiction is resolved upon consideration of the accelerations of the zonal mean wind by baroclinic eddies of finite amplitude, detailed in the next section. It should be noted that comparison between weakly nonlinear accelerations to the zonal mean jet by the most unstable normal mode, and the fully nonlinear accelerations reported in the next section, are independent of the form of the perturbation in the nonlinear experiments.

\section{The fully nonlinear problem}

A natural extension of the previous section which allows us to investigate the nonlinear effects on the baroclinic development is to perform life cycle experiments, using the same initial conditions as before. Our approach here is similar to that of our previous study (W04), but here we vary only the stratospheric shear, and perform life cycles at zonal wavenumbers other 

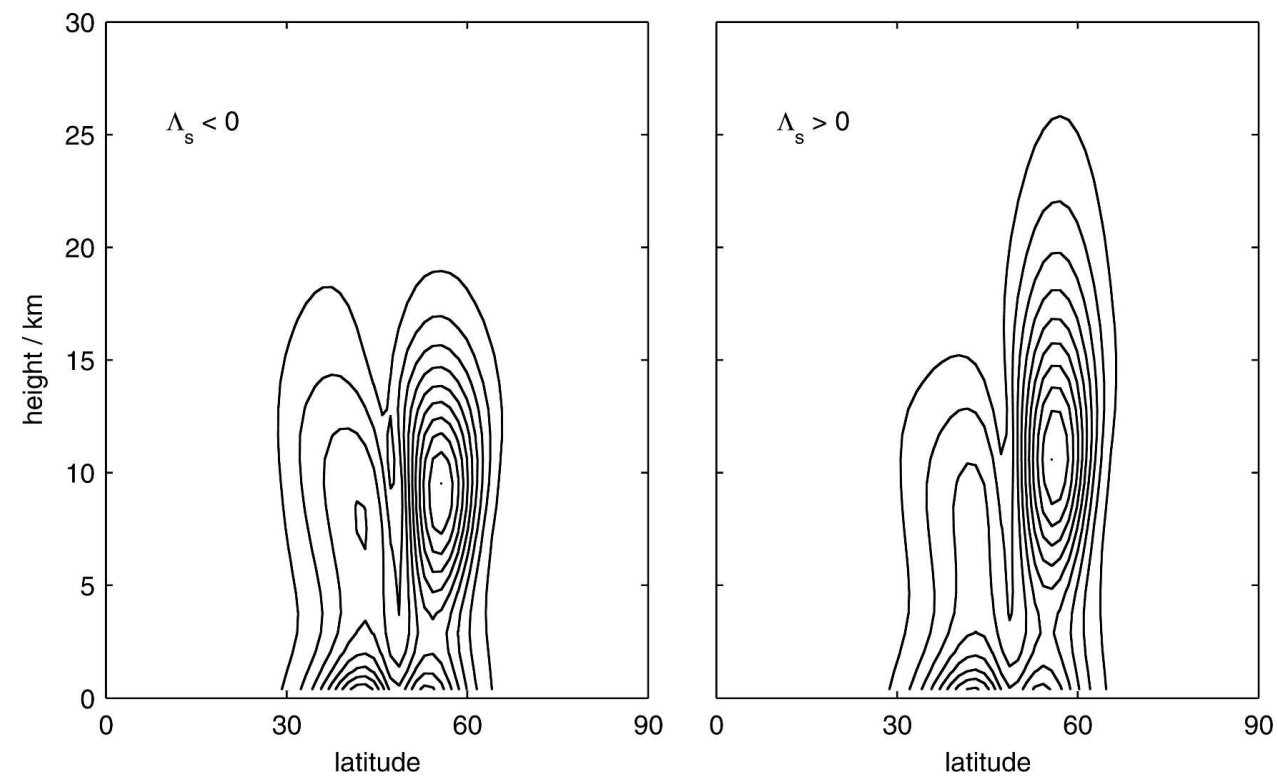

FIG. 5. The structure of the amplitude of the perturbation zonal wind for the normal modes at wavenumber 7. (left) The negative stratospheric shear case and (right) the positive stratospheric shear case. In each panel, the contour interval is $1 / 10$ th of the maximum value of the perturbation amplitude.

than 6. We solve initial value problems with the fully nonlinear primitive equations, and consider the effect of the life cycles, with different stratospheric shears and different wavenumbers, on the mean flow. By considering the surface geopotential height we can relate our results to the observational work cast in the annular mode framework.

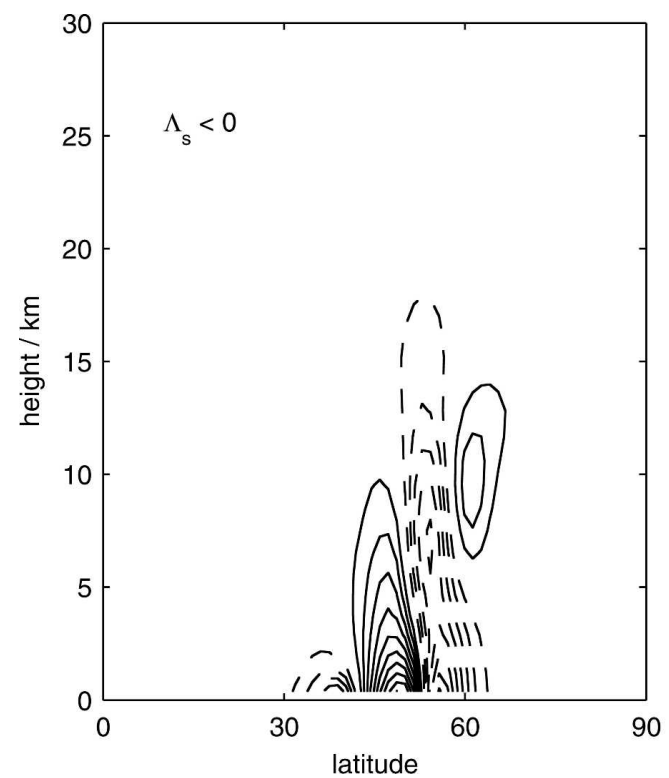

\section{a. Details of the calculation}

We use the unmodified, fully nonlinear version of $\mathrm{BOB}$ with the same initial conditions and initial perturbation as in section 3, again at T85 spectral resolution and 40 pressure levels. A horizontal hyperdiffusion $\nabla^{6}$ is used, with a time scale of $1 \mathrm{~h}$ at the shortest resolved

FIG. 6. The acceleration of the zonal mean zonal wind by the normal modes at wavenumber 7. (left) The negative stratospheric shear case and (right) the positive stratospheric shear case. The contour interval is $1 / 10$ th of the maximum acceleration, and dashed lines represent negative (westward) accelerations. 

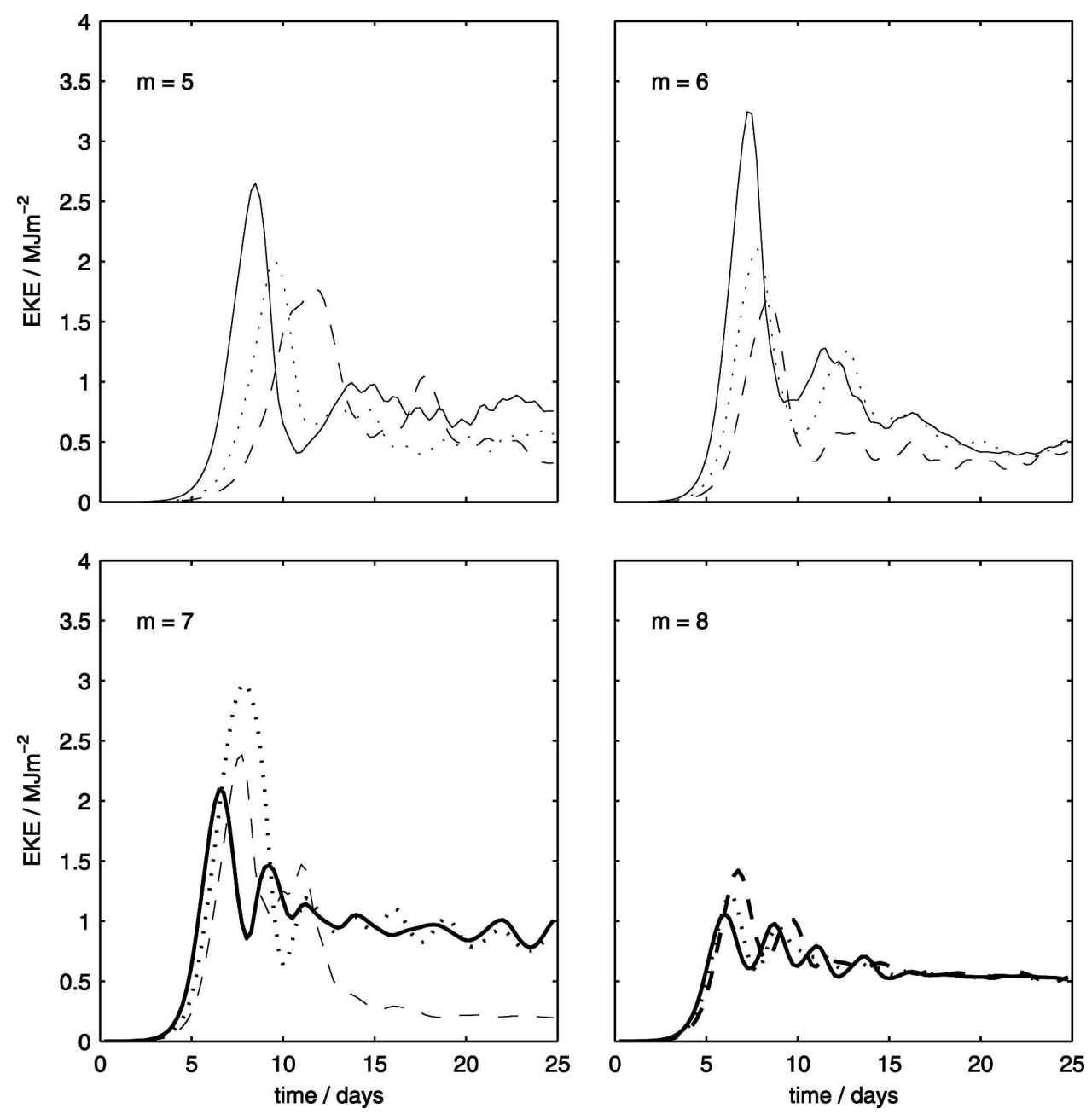

FIG. 7. Eddy kinetic energy for life cycles at wavenumbers 5 to 8. Dashed, dotted, and solid lines represent negative, zero, and positive stratospheric shear, respectively, and LC2 (cyclonic breaking) cases are represented by bold lines.

length scale. Our results are qualitatively insensitive to variations in this time scale, and they are qualitatively unchanged at spectral resolutions T42 and T170. Sensitivity to the form of the initial perturbation, the cutoff height, and the widths of the corners of the wind and temperature vertical profiles was also investigated, and no qualitative effect on our results was found.

\section{b. LC1/LC2 transition}

Figure 7 shows the EKE of life cycles with initial perturbations of wavenumbers 5 to 8 , close to the wavenumbers at which the linear growth rate peaks, and close to the peak in the observed power spectrum of the atmosphere, of approximately wavenumber 6 (Pierrehumbert and Swanson 1995). Each panel shows a different wavenumber perturbation, and the dashed, dotted, and solid lines are cases with negative, neutral, and positive stratospheric shear, respectively. The different growth rates predicted by the linear calculations above are evident, and there are two additional points of note. At wavenumbers 5 and 6 , and for the two cases with the lowest stratospheric shear at wavenumber 7 , saturation EKE increases monotonically with stratospheric shear. For the positive shear case at wavenumber 7 , and all cases at wavenumber 8 , the saturation amplitude is much reduced, and decreases with stratospheric shear. For these cases EKE persists at a large fraction of its peak value. The persistence of EKE, and the change in dependence on stratospheric shear suggests a fundamental change in behavior. From T93 we know that persistent EKE is associated with LC2 or anomalous life cycles, in which the wave breaking at the tropopause following the saturation of the instability is toward the pole, leaving large-scale, persistent PV anomalies. The EKE for the LC2 cases is plotted with bold lines. 
day 8
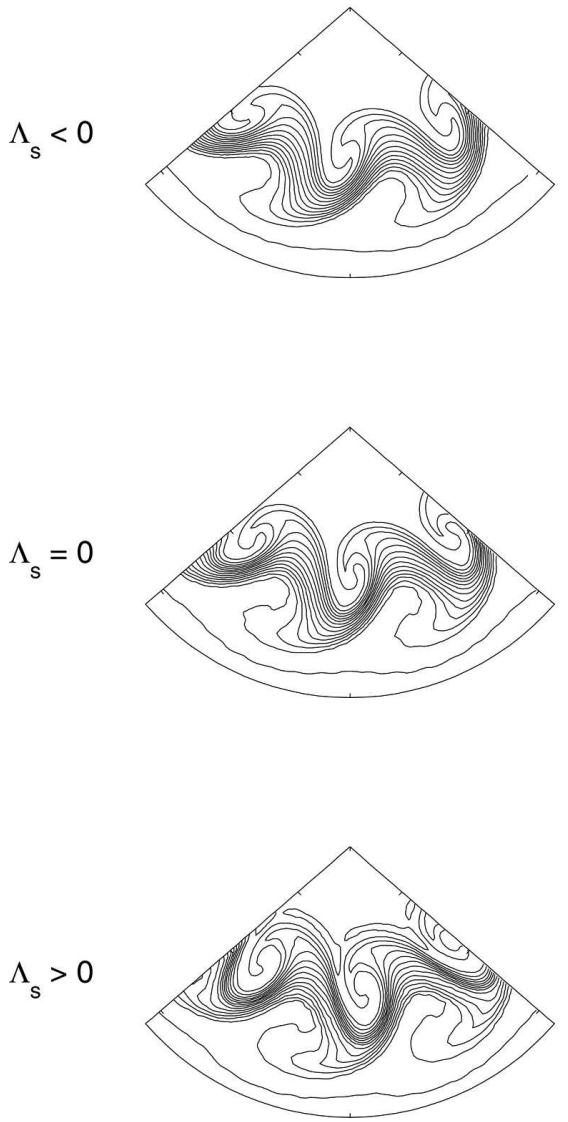

day 9
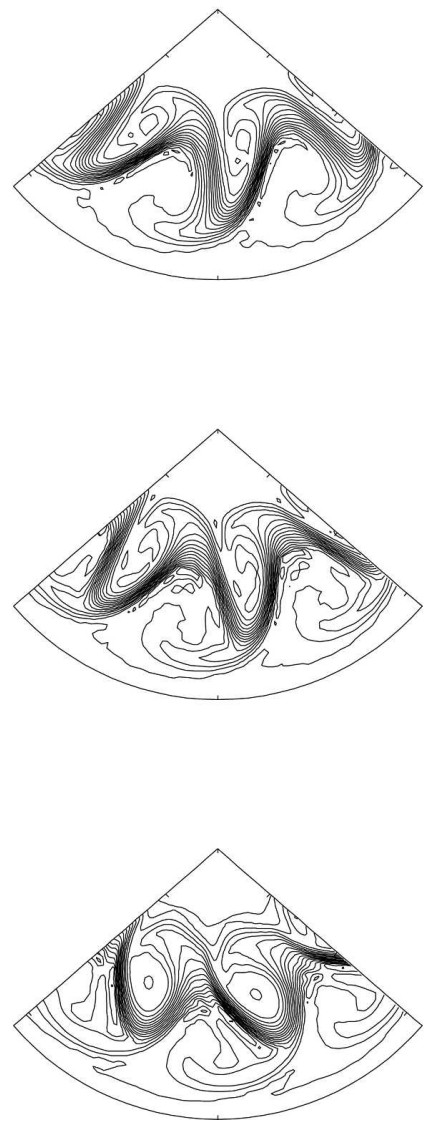

day 10
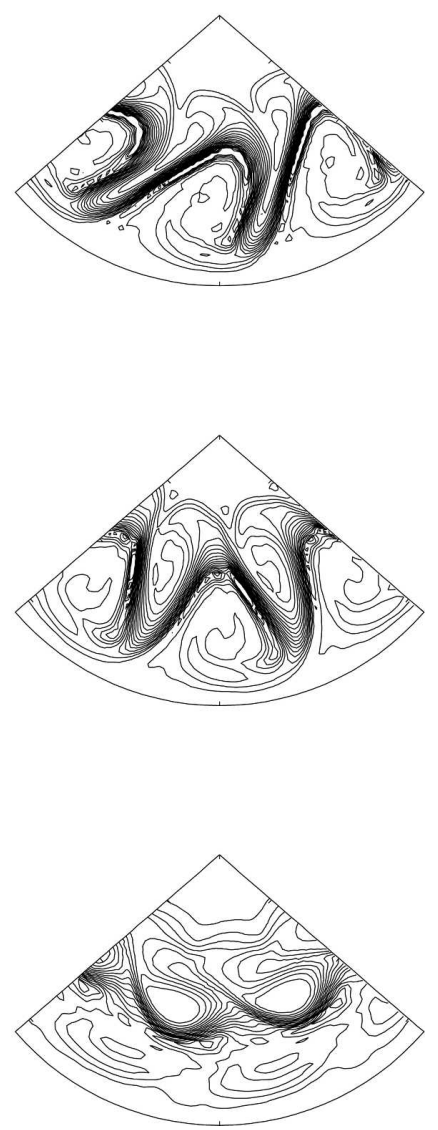

FIG. 8. Potential vorticity on $315-\mathrm{K}$ isentrope on three successive days for the life cycle perturbed with wavenumber 7 for the three values of stratospheric shear.

To investigate whether a change in life cycle type has occurred, we plot PV on the $315-\mathrm{K}$ isentrope, shown in Fig. 8. This isentropic surface lies between about $5 \mathrm{~km}$ on the equatorward side of the jet, and $14 \mathrm{~km}$ on the poleward side. We show PV on $315-\mathrm{K}$ instead of the potential temperature on 2-PVU surface plotted in T93 since PV may be a multivalued function of height, and the features that distinguish cyclonic and anticyclonic wave breaking are apparent in both quantities. In all three cases with a wavenumber-7 perturbation, the wave breaks both poleward and equatorward at day 6 , but by day 8 there is a clear, qualitative difference between the negative and the positive stratospheric shear cases. With negative stratospheric shear, the wave breaks equatorward, irreversibly deforming the PV contours and allowing the eddy kinetic energy to cascade to short length scales where it is removed by diffusion. With positive stratospheric shear the equatorward breaking is inhibited, and the wave breaks poleward, forming persistent large-scale PV anomalies that do not dissipate. These two behaviors, hall- marks of $\mathrm{LC} 1$ and $\mathrm{LC} 2$, confirm that it is possible to change the type of life cycle by varying stratospheric shear alone.

Hartmann and Zuercher (1998, hereafter HZ98) previously investigated the transition from LC1- to LC2type behavior by adding barotropic shear to the equatorward side of a tropospheric jet similar to ours. As the added barotropic shear increased, the location of the critical surface on the equatorward side of the jet at which the phase speed of the waves equaled the speed of the background flow, and at which nonlinear Rossby wave breaking occurred, moved farther toward the equator. Thus the waves were trapped in a reflecting cavity and persisted (hence the persistence of eddy kinetic energy in LC2). In HZ98, the critical value of barotropic shear occurred when the growing wave, whose small-amplitude effect on the jet was to cause deceleration to the north and south of its maximum, could not remove enough of the barotropic shear to allow subsequent wave breaking to occur. Thus, the change from LC1- to LC2-type behavior was ascribed 
to the latitude of the critical surface, which moved toward the equator as barotropic shear was added.

HZ98 also found that at wavenumbers 8 and above all values of barotropic shear resulted in life cycles that were of LC2 type, and below wavenumber 4 all life cycles were of LC1 type. With our jet specification we see a transition from LC1 to LC2 only at wavenumber 7, which suggests that baroclinic development is less sensitive to changes in the stratosphere than to changes in the troposphere.

In this study we see the change from LC1- to LC2type behavior in what is, below the tropopause, effectively the same jet. Figure 9 shows the location of the critical surfaces $\bar{u}=c$ for the three cases of different stratospheric shear at wavenumber 7 and day 6, a similar time before the peak EKE as in HZ98. There is a suggestion that the critical line on the equatorward side of the jet moves poleward as the shear increases and the life cycle changes from LC1 to LC2, but it is by no more than $1^{\circ}$ of latitude between 5 and $8 \mathrm{~km}$. This small poleward shift is due to the increase in phase speed of the growing mode with increasing stratospheric shear, since the zonal mean jet is the same below the tropopause. This result is difficult to reconcile with the argument of HZ98 and T93, and suggests that the location of the equatorward critical surface alone does not determine the life cycle type. The critical surface location may simply be a consequence of changing the basic state jet. Randel and Held (1991) find that Rossby waves breaking toward the equator break at $10^{\circ}$ to $20^{\circ}$ poleward of the critical surface, and that waves with lower phase speeds penetrate farthest equatorward. Our life cycle results agree with this: low phase speed waves, corresponding to low stratospheric shears, develop into LC1 life cycles with wave breaking toward the equator, and the higher phase speed waves, corresponding to high-stratospheric shear, break toward the pole as LC2 life cycles. It should be noted that even though the difference in phase speed resulting from changes to the stratospheric shear are small—of the order of $2 \mathrm{~m} \mathrm{~s}^{-1}$ - the LC1/LC2 transition occurs when initial conditions on two sides of a separatrix in phase space evolve away from each other, so that nearly indistinguishable initial conditions may give rise to completely different nonlinear evolutions. The sharpness of the transition induced by changing barotropic shear on the equatorward side of the jet has already been documented in HZ98.

Experiments with incrementally varying stratospheric shears show that there is a sharp transition at zonal wavenumber 7 from LC1 to LC2, and this transition occurs at values of stratospheric shear between -0.5 and $0 \mathrm{~m} \mathrm{~s}^{-1} \mathrm{~km}^{-1}$. This value of shear is within the

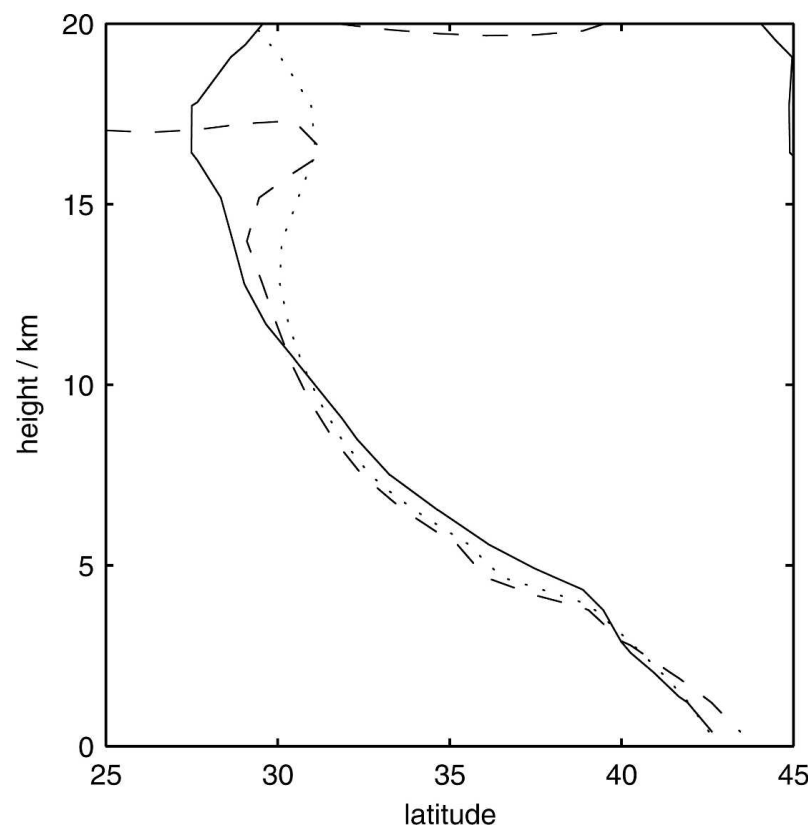

FIG. 9. Location of critical surfaces $(u=c)$ at day 5 of the life cycle integrations with initial perturbation of wavenumber 7 . Dashed, dotted, and solid lines represent negative, zero, and positive stratospheric shear, respectively.

range of observed $100-\mathrm{hPa}$ shears and, although the transition occurs at values of stratospheric shear that are somewhat numerically sensitive, its occurrence is robust. It may thus be possible to find the same dependence of life cycle type on stratospheric shear in the observations, given a suitable algoriT93 for diagnosing cyclonic and anticyclonic wave breaking.

\section{c. Life cycle results in the annular mode framework}

The transition from LC1 to LC2 is the most dramatic consequence of changing stratospheric shear, but it occurs only at one wavenumber, and its actual occurrence in the atmosphere is untested. We therefore return to consideration of the tropospheric annular mode response to stratospherically modulated baroclinic life cycles.

Changes in the annular mode index for a zonally symmetric tropospheric jet correspond mostly to changes in the latitude of the jet (Wittman et al. 2005), but the annular mode is usually defined as the first EOF of geopotential height. Since our model lacks a prognostic equation for the surface value of geopotential height, following W04, we instead calculate the zonal mean surface geopotential height $\bar{Z}_{\text {surf }}$ from the $v$-momentum Eulerian mean equation (see, e.g., Andrews et al. 1987), neglecting the vertical terms, which are small.

Since we cannot statistically derive an annular mode function from a large series of observations, as we could if we were considering reanalysis data or output from a 

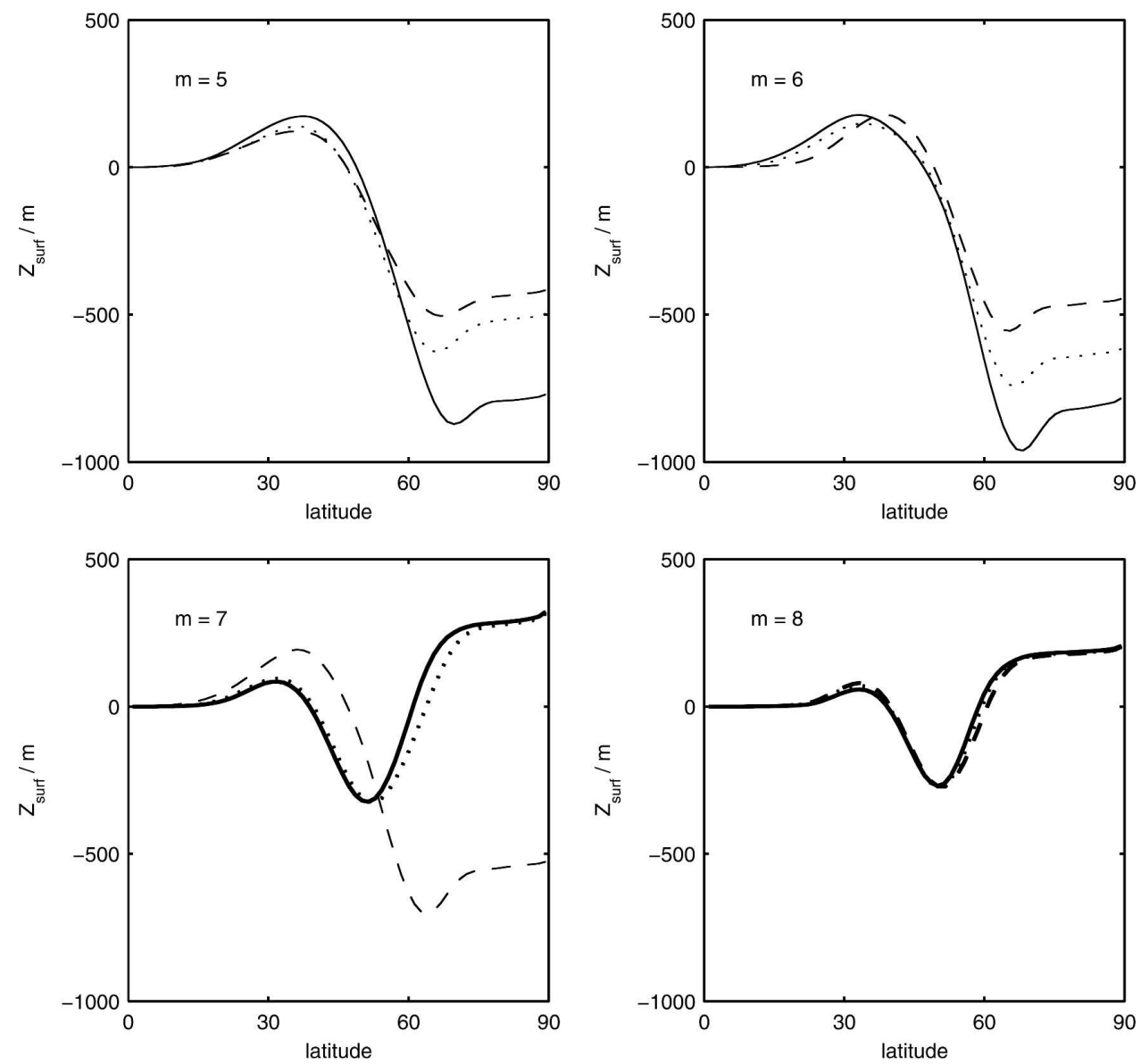

FIG. 10. Time-mean surface geopotential height for life cycles at wavenumbers 5 to 8 . Dashed, dotted, and solid lines represent negative, zero, and positive stratospheric shear, respectively.

long numerical integration, for this section our strategy will be to approximate the annular mode as a step function of latitude in surface geopotential, crossing zero at the latitude of the jet maximum:

$$
\operatorname{AM}(\phi)=\left\{\begin{array}{cc}
1 & \phi<45^{\circ} \mathrm{N} \\
-1 & \phi>45^{\circ} \mathrm{N}
\end{array} .\right.
$$

Thus, we can calculate the zonal mean zonal wind, calculate the zonal mean surface geopotential height $\bar{Z}_{\text {surf }}$, and project onto the annular mode function and integrate with time to arrive at an annular mode index (AMI) by which to quantify the dependence of the meridional evolution of the jet during baroclinic instability:

$$
\operatorname{AMI}(t)=\int_{\tau=0}^{t} \int_{\phi=0}^{90^{\circ} \mathrm{N}} \bar{Z}_{\text {surf }}(\phi, \tau) \operatorname{AM}(\phi) d \phi d \tau .
$$

The position of the jet, and the annular mode index, are fixed, in the atmosphere, by a balance between forcing by baroclinic eddies and solar forcing. The solar forcing tends to establish a jet at an equilibrium posi- tion in midlatitudes, baroclinic eddies drive the jet away from this position, and solar forcing returns the jet to its original position. Our unforced life cycle experiments lack the restoring solar heating, and so we are only able to measure how far the jet is shifted from its original position by baroclinic eddies. The resulting shift, however, is suggestive of how the position of the jet responds to changing stratospheric conditions, since the time scale of the life cycle (saturation occurs after about 5 days) is much shorter than that of the restoring solar forcing [Held and Suarez (1994) uses a radiative cooling time scale of 40 days outside the Tropics]. Comparing AMI between life cycles therefore gives a measure of the effect of baroclinic eddies, modulated by different stratospheric shears, on the annular mode index. It is perhaps worth clarifying that projecting $\bar{Z}_{\text {surf }}$ onto the annular mode, and then comparing between life cycles, is different from our approach in W04, where we projected the difference in $\bar{Z}_{\text {surf }}$ between two life cycles onto the annular mode, and identified the resulting quantity as a measure of the surface response to 

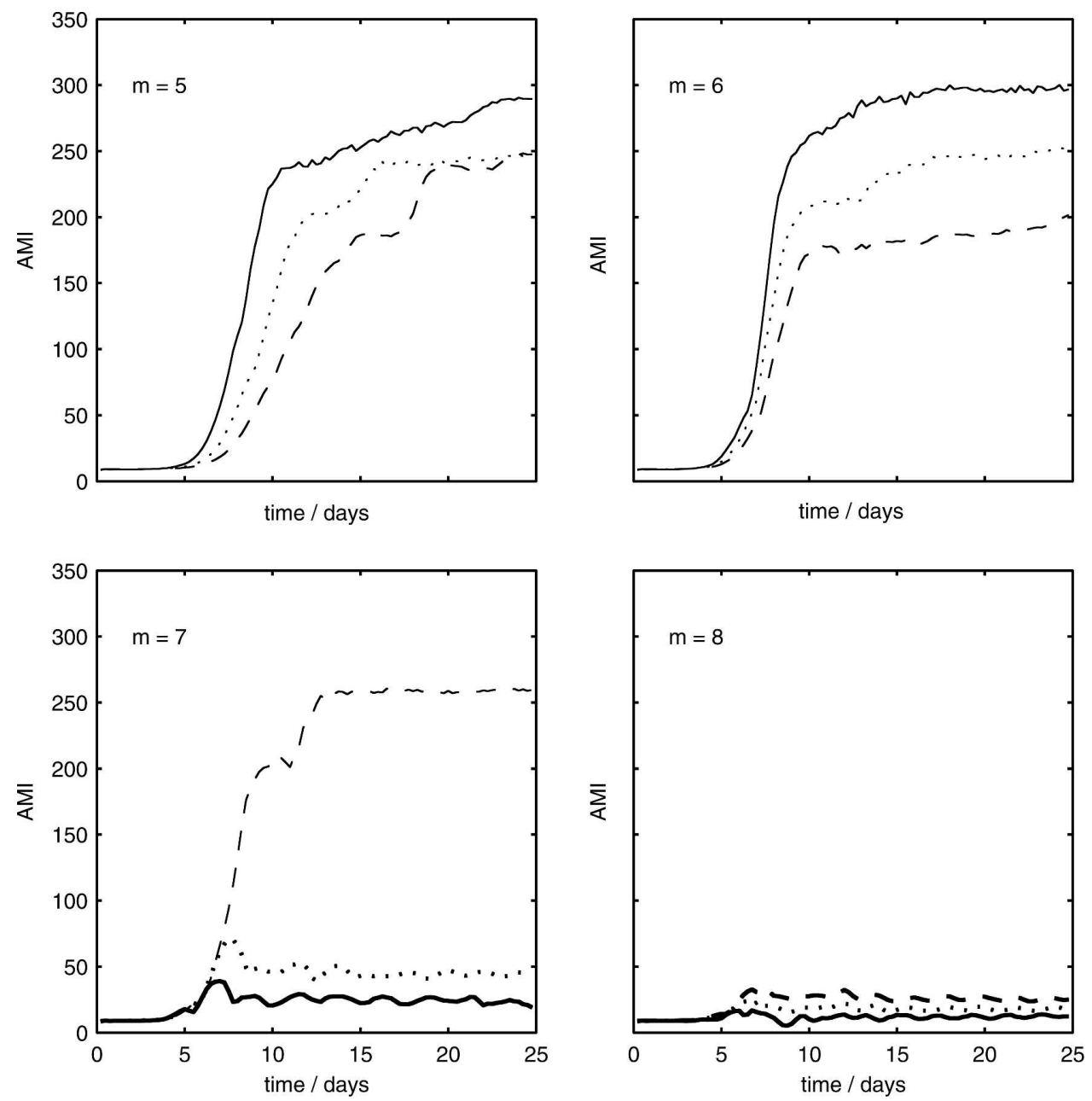

FIG. 11. Instantaneous projection of $Z_{\text {surf }}$ onto the synthetic annular mode function for life cycles at wavenumbers 5 to 8 . Dashed, dotted, and solid lines represent negative, zero, and positive stratospheric shear, respectively.

changes in the stratosphere. Here, because $\bar{Z}_{\text {surf }}$ changes dramatically between LC1 and LC2 life cycles, we project the raw quantity.

Figure 10 shows the time mean of $\bar{Z}_{\text {surf }}$ over the 25day duration of the life cycles. In the LC1 regime, increasing stratospheric shear leads to a deepening of the poleward branch of the surface geopotential, corresponding to a greater poleward strengthening of the zonal mean jet as a result of the instability, and projecting more strongly onto the annular mode. In the LC2 regime, changing the stratospheric shear has little effect on the surface geopotential height.

Figure 11 shows AMI, the cumulative projection of the surface geopotential height onto the annular mode. For all of the LC1 cases the signal grows rapidly as the instability approaches its peak amplitude. The ordering of the growth rates and final values of this index are the same as the growth rates and peak values of the EKE, and the final separation in the values of the projection coefficient is established at around the time of maximum EKE.

For the LC2 cases, the growth of the projection coefficient is initially comparable with that of the LC1 cases, but slows rapidly before the instability saturates, and remains at a small fraction of its value in the $\mathrm{LC} 1$ cases. It is also apparent that for the LC1 cases, increasing stratospheric shear increases AMI at all times, and that AMI rises most strongly just as the life cycles saturate.

To establish how this annular mode signal is produced, and what produces the differences between cases of different stratospheric shears, it is instructive to examine the total acceleration of the zonal mean zonal wind by life cycles with different stratospheric shears. The total change over the 25-day life cycle in zonal mean zonal wind for wavenumber 6 (all LC1 type) is shown in Fig. 12. As stratospheric shear is increased 

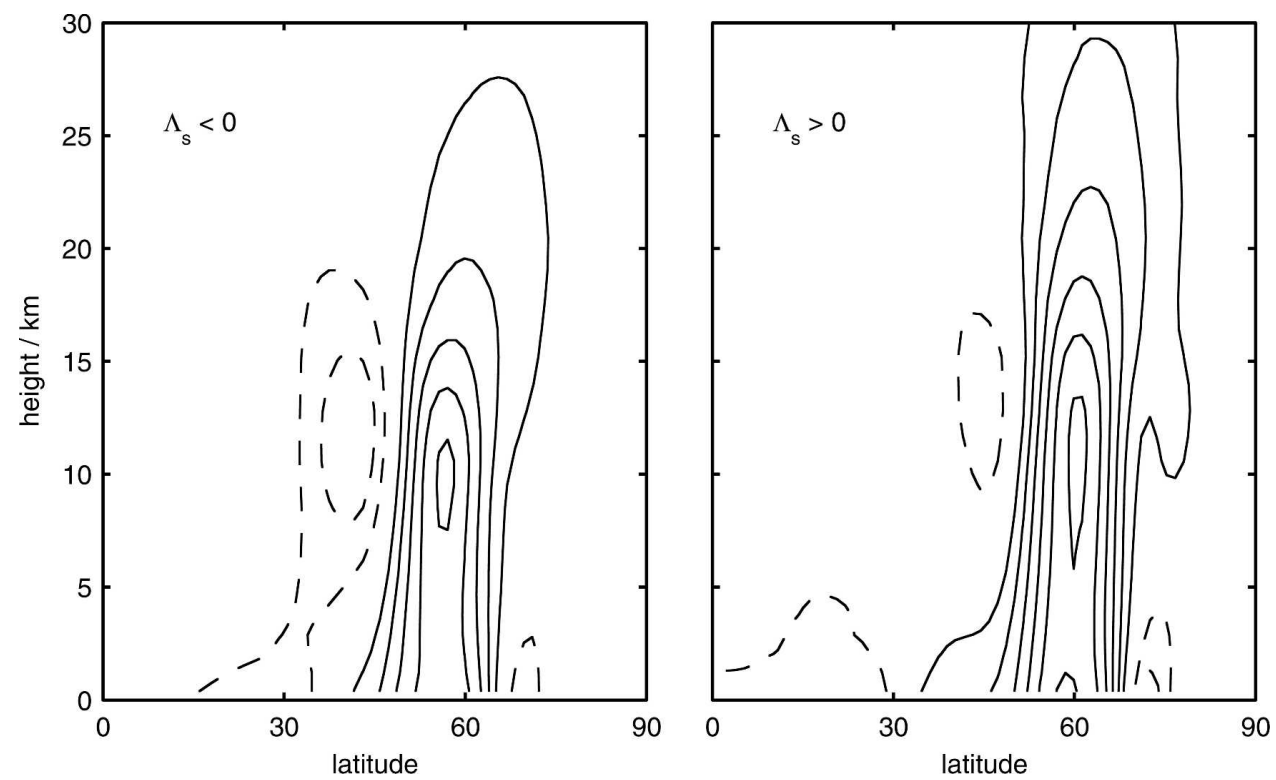

FIG. 12. Total acceleration of the zonal mean zonal wind by the life cycle at wavenumber 6 for the negative and positive stratospheric shear cases. The contour interval is $10 \mathrm{~m} \mathrm{~s}^{-1}$, and negative contours are dashed.

from -4 to $+4 \mathrm{~m} \mathrm{~s}^{-1} \mathrm{~km}^{-1}$, the total acceleration of the jet at the surface on the poleward side increases from 40 to $60 \mathrm{~m} \mathrm{~s}^{-1}$, and the location of the maximum acceleration remains unchanged at about $60^{\circ} \mathrm{N}$. The weaker deceleration on the equatorward side of the jet is correspondingly decreased with increasing stratospheric shear from 20 to $10 \mathrm{~m} \mathrm{~s}^{-1}$, also remaining in the same place. Thus increasing stratospheric shear produces a stronger annular mode signal by causing a greater acceleration of the jet, so that the poleward-displaced jet resulting from a baroclinic life cycle becomes stronger, and the increasing annular mode response to increasing stratospheric shear is a consequence of the more rapid growth and larger saturation amplitude that occur with increased stratospheric shear.

For the wavenumber-7 cases, shown in Fig. 13, which show the transition from LC1 to LC2 with increasing stratospheric shear, it is apparent that the resultant acceleration of the jet reverses sign at the transition, so that the LC2 life cycle moves the jet equatorward instead of poleward. For higher wavenumbers, all in the LC2 regime, the maximum acceleration is then only slightly sensitive to the stratospheric shear.

Several observational papers (e.g., Hartmann et al. 2000) have found a connection between high stratospheric annular mode indices and increased equatorward Eliassen-Palm (EP) flux in the region of the tropopause, corresponding to increased poleward momentum transport and poleward jet displacements. The EP fluxes in our life cycles, shown in Fig. 14, show the same dependence on stratospheric shear, with high- stratospheric shear giving rise to enhanced equatorward EP flux at the tropopause. This EP flux pattern, which is similar for both values of stratospheric shear, is that of a typical LC1 life cycle and increasing stratospheric shear increases the amplitude the instability reaches before saturating, so that the effect of increasing stratospheric shear is just to increase the amplitude of the LC1 EP flux pattern. The increased penetration of EP fluxes into the stratosphere with higher stratospheric shear is consistent with the normal mode extending farther into the stratosphere.

In summary, for low wavenumbers (below 6), life cycles are LC1 type, with wave breaking on the equatorward side of the jet. As stratospheric shear is increased, the saturation amplitude of the growing eddies increases, giving rise to larger total accelerations that displace the jet farther poleward, and result in larger annular mode signals. At wavenumber 7, increasing stratospheric shear changes the life cycle from an LC1 to an LC2, possibly by changing the phase speed, and thereby the location of the critical surface on the equatorward side of the jet. The LC2 cases show little sensitivity to stratospheric shear, and all life cycles at wavenumbers higher than 7 are of an LC2 type.

\section{Observations}

The above life cycle experiments suggest that for the lower synoptic wavenumbers, stratospheric shear is positively correlated with the annular mode index in the troposphere, and vice versa. To establish whether a 

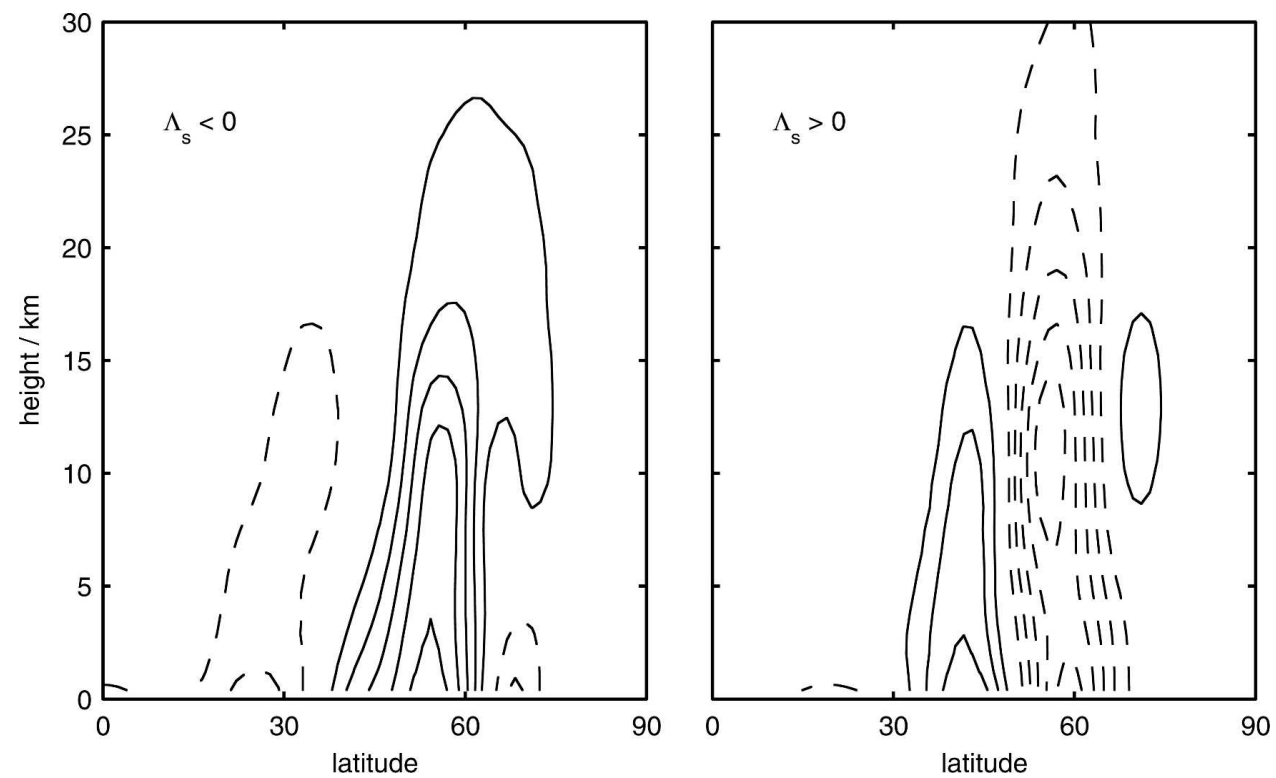

FIG. 13. Total acceleration of the zonal mean zonal wind by the life cycle at wavenumber 7 for the negative and positive stratospheric shear cases. The contour interval is $10 \mathrm{~m} \mathrm{~s}^{-1}$ and negative contours are dashed.

similar relationship between stratospheric shear and tropospheric annular mode index can be found in the observations, we examine National Centers for Environmental Prediction-National Center for Atmospheric Research (NCEP-NCAR) data (Kalnay et al. 1996), for which the daily northern annular mode index has been calculated at each pressure level (Baldwin and Dunkerton 2001, hereafter BD01). Approximately 6000 wintertime values of the NAM index are categorized

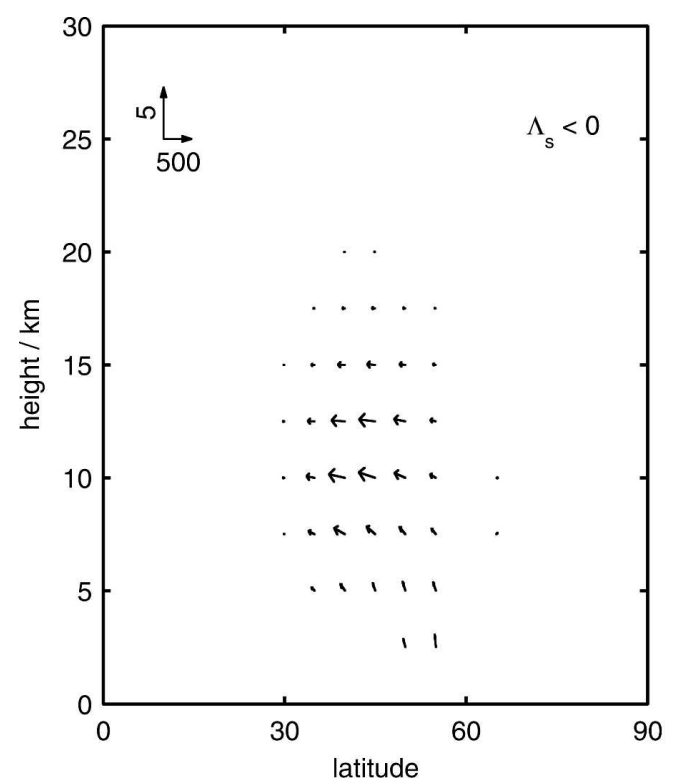

into three approximately equally sized groups by the anomaly of the wind shear at $100 \mathrm{hPa}$. Figure 15 shows the mean NAM index as a function of pressure for days with negative, neutral, and positive stratospheric shear anomalies, and the probability distributions of the $1000-\mathrm{hPa}$ NAM index for the three categories of shear anomaly.

The probability distribution functions (PDFs) of 1000-hPa NAM index, $p(x)$, are constructed using a Gaussian kernel:

FIG. 14. The EP fluxes for wavenumber-6 life cycles, for the cases with negative and positive stratospheric shear, at peak EKE (days 8 and 7, respectively). The arrows in the top-left corners give the scale $\left(\mathrm{m}^{2} \mathrm{~s}^{-2}\right)$. 

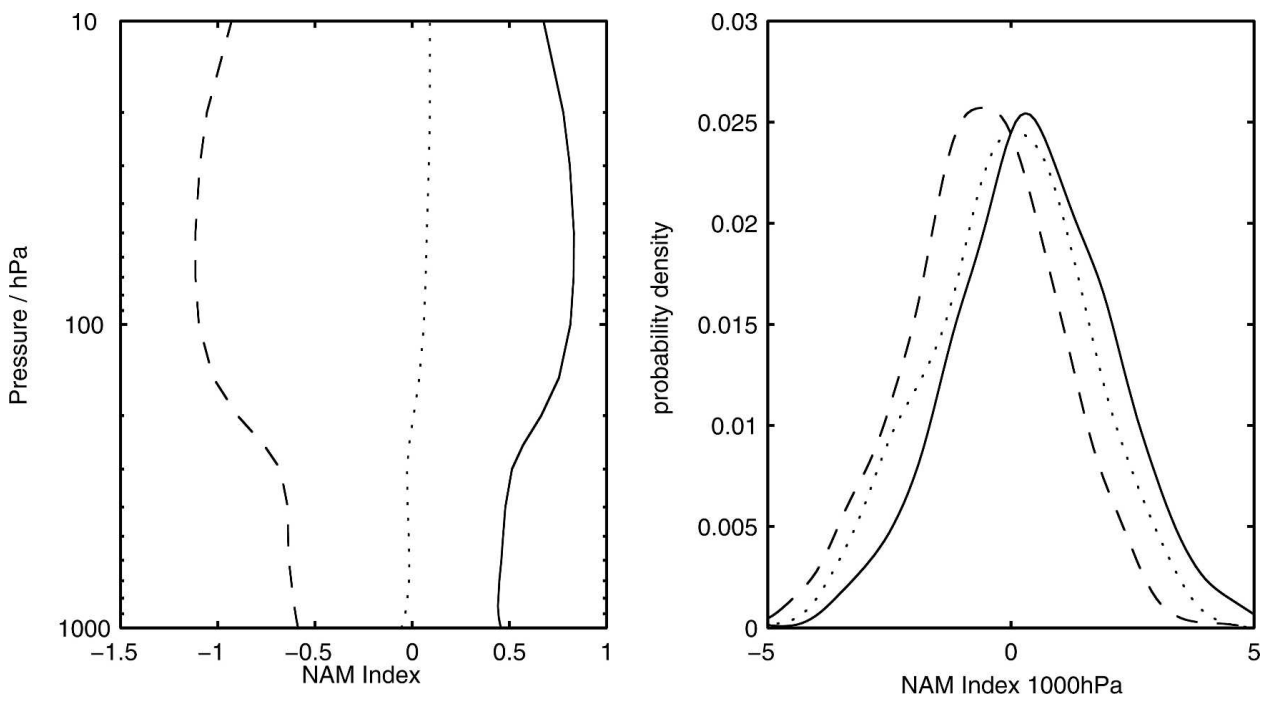

FIG. 15. (left) NAM index as a function of pressure for different stratospheric shear anomalies. (right) Surface NAM PDFs for negative, neutral, and positive stratospheric shear categories. In both panels dashed, dotted, and solid lines represent negative, zero, and positive stratospheric shear, respectively.

$$
p(x) \propto \sum_{i} \exp \left(-\frac{x-y_{i}}{2 h^{2}}\right),
$$

where $i$ is over all observations, $y_{i}$ are the observed NAM values, and $h$ the width of the kernel, given by (Silverman 1986) $1.06 \sigma n^{-1 / 5}$, where $\sigma$ is the population standard deviation, and $n$ is the number, of all of the NAM observations in each stratospheric shear category.

From the distributions, it is evident that the mean NAM index shifts by about 1 between the high- and low-stratospheric shear cases, comparable to the shift in NAM index between strong vortex and weak vortex events in BD01. It is also worth noting that their weak vortex events were defined as stratospheric NAM indices of less than -3 standard deviations from the mean, and the strong events had stratospheric NAM indices of above 1.5 standard deviations. Assuming a roughly normal distribution of stratospheric shears, our strong shear cases are those with shears greater than about 0.5 standard deviations, and our negative shear cases have shears less than about -0.5 standard deviations. Thus stratospheric shear appears to be a more reliable predictor of surface annular mode index than stratospheric annular mode index.

The above observational study shows that the atmosphere qualitatively behaves in a way which is at least consistent with the response to shear in life cycle experiments. A more direct and quantitative comparison between the life cycle experiments and observations may be made by comparing the change in uppertropospheric momentum fluxes accompanying changes in shear in the life cycle experiments, and in observations.
Limpasuvan et al. (2004, their Fig. 3, left-hand column) shows the evolution of zonal mean zonal wind during a composite stratospheric sudden warming (SSW). At the peak of the SSW, the maximum lower stratospheric shear anomaly is about $-1 \mathrm{~m} \mathrm{~s}^{-1} \mathrm{~km}^{-1}$. The accompanying maximum anomaly in synoptic momentum flux $F_{y}$ integrated between $50^{\circ}$ and $90^{\circ} \mathrm{N}$ (Limpasuvan et al. 2004, their Fig. 10e), is about $30 \times 10^{6}$ $\mathrm{kg} \mathrm{s}^{-2}$. In our life cycle experiments a change of -4 $\mathrm{m} \mathrm{s}^{-1} \mathrm{~km}^{-1}$ in lower stratospheric shear gives rise to a change of $70 \times 10^{6} \mathrm{~kg} \mathrm{~s}^{-2}$ in the 300 -hPa momentum flux integrated between $50^{\circ}$ and $90^{\circ} \mathrm{N}$ (Fig. 16), which, assuming a linear response, gives about a change of about $18 \times 10^{6} \mathrm{~kg} \mathrm{~s}^{-2}$ in response to a change of -1 $\mathrm{m} \mathrm{s}^{-1} \mathrm{~km}^{-1}$ in lower stratospheric shear. Hence the response to a unit change in shear of upper-level synoptic momentum fluxes in life cycles is about two-thirds the size of the response of the observed atmosphere, which, considering the highly idealized nature of the life cycle experiments, is not a bad agreement.

We may also note that Limpasuvan gives a surface NAM response of about -0.75 to a $-1 \mathrm{~m} \mathrm{~s}^{-1} \mathrm{~km}^{-1}$ change in stratospheric shear, and, while we cannot calculate a meaningful NAM index in our life cycle experiments, the change in NAM index reported by Limpasuvan in the observations is of the same sign as in our life cycles, and is significant.

In summary, the correspondence between tropospheric NAM index and lower stratospheric shear suggested by the life cycle experiments is corroborated by the NCEP-NCAR reanalysis data, with increased lower stratospheric shear associated with higher mean 


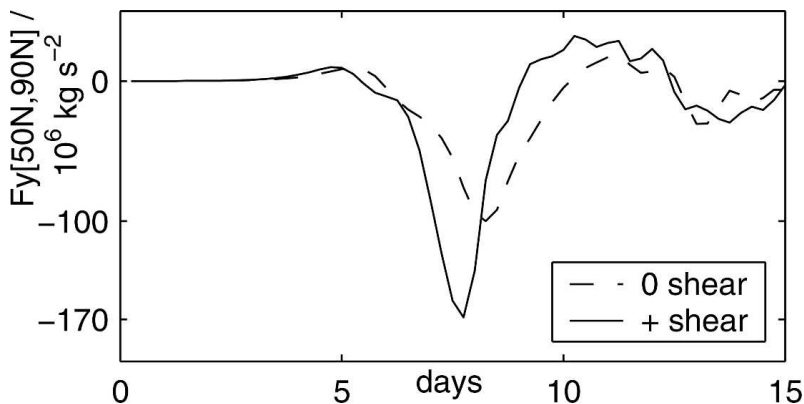

FIG. 16. Momentum fluxes at $300 \mathrm{hPa}$ integrated between $50^{\circ}$ and $90^{\circ} \mathrm{N}$ for the positive $(+)$ shear and 0 shear wavenumber- 6 life cycles.

values of the annular mode index at all heights, and vice versa. The response to tropospheric momentum fluxes, by which the zonal mean jet structure is changed and the NAM signal established, is comparable in both life cycles and observations.

\section{Conclusions}

We have found that baroclinic development is affected by stratospheric shear, and confirmed robustly that stratospheric shear can account for the connection between the stratospheric and tropospheric NAM signals previously reported (Wittman et al. 2004).

Solving the linear instability problem in $3 \mathrm{D}$ primitive equations, we have found that the effect of stratospheric shear on the growth rate and phase speed is qualitatively the same as in a simple Eady-like 1D model, we have determined the meridional structure of the most unstable modes, and their dependence on stratospheric shear. Life cycle experiments have shown that changing stratospheric shear can induce a transition between LC1 and LC2 life cycles at zonal wavenumber 7. In the annular mode framework, we have found that, for LC1 life cycles, increasing stratospheric shear increases the poleward displacement of the jet by the finite-amplitude baroclinic eddies; this agrees with the observed stratosphere-troposphere NAM relationship. The change in acceleration of the zonal mean jet by baroclinic life cycles is a result of the saturation amplitude increasing with stratospheric shear-the larger the baroclinic wave grows before breaking, the greater the effect on the mean flow. Examining the connection between NAM index and 100-hPa shear in reanalysis data, we have found that higher stratospheric shears are, in fact, associated with higher tropospheric NAM indices, and vice versa.

Although we have shown that lower stratospheric shear can produce a tropospheric NAM signal by modulating baroclinic eddies, this does not rule out other mechanisms by which the stratosphere may influ- ence the tropospheric circulation-indeed, it would be surprising if shear-modulated eddies were the only mechanism responsible for the downward propagation of NAM anomalies from the stratosphere to the troposphere. In particular, recent work (Kushner and Polvani 2004; Song and Robinson 2004; Thompson et al. 2006) suggests that downward control may also be involved in producing surface NAM responses to stratospheric disturbances. Though it may be difficult to design a clean numerical experiment that would ascribe causality uniquely to any one mechanism, or exclude any other, the weight of evidence suggests that baroclinic eddies are at least one component of the downward propagation of NAM anomalies to the surface. It should also be noted that the amplitude of the tropospheric response to changes to the stratosphere is small: as we reported in W04 tropospheric responses to changes to the tropospheric mean flow are at least 10 times larger than responses to the stratosphere, and, as such it surely remains difficult to fully disentangle what tropospheric behavior is a response to the stratosphere and what behavior constitutes internal variability. However, we have demonstrated and elucidated a plausible linkage between the stratosphere and troposphere.

All previous life cycle studies have induced transitions between LC1 and LC2 behavior by changing the structure of the jet in the troposphere, and Hartmann (2000) found explicitly that the transition could be induced by adding equatorward barotropic shear only if the shear was added near the ground. The results presented here suggest that the evolution of synoptic eddies may be affected by conditions in the lower stratosphere. By developing an objective algoriT93 to classify midlatitude wave breaking events into cyclonic and anticyclonic categories, one may be able to determine whether stratospheric shear is correlated to the prevalence of LC1 and LC2 type events in the observations, confirming the stratospheric shear-driven LC1/LC2 transition which we have found in these life cycle experiments. Such an algoriT93 would also find uses beyond the issue of stratosphere-troposphere coupling: LC1/LC2 wave breaking has been implicated in lowfrequency internal tropospheric variability (e.g., Hartmann and Zuercher 1998).

In a wider context, this work adds to the growing body of evidence on the importance of the stratosphere in climate modeling and weather forecasting. A consistent poleward shift of the storm tracks has been documented in the recent Intergovernmental Panel on Climate Change (IPCC) simulations of twenty-first century climate (Yin 2005; Miller et al. 2006). the results presented here, showing that lower stratospheric shear 
is able to directly impact synoptic eddies (and thus the location of the midlatitude jets), suggest that a better representation of the lower stratosphere in climate models might be important. Similarly, greater attention to the lower stratosphere (e.g., in terms of higher vertical resolution about the tropopause) might help in improving weather forecasts, as suggested by Baldwin et al. (2003).

Acknowledgments. This research was funded, in part, by an award to the Cooperative Institute for Climate Applications and Research (CICAR) from the U.S. National Oceanic and Atmospheric Administration and, in part, from a grant to Columbia University from the U.S. National Science Foundation.

\section{REFERENCES}

Andrews, D. G., J. R. Holton, and C. B. Leovy, 1987: Middle Atmosphere Dynamics. Academic, 489 pp.

Baldwin, M., and T. Dunkerton, 1999: Propagation of the Arctic Oscillation from the stratosphere to the troposphere. J. Geophys. Res., 104, 30 937-30946.

—, and —, 2001: Stratospheric harbingers of anomalous weather regimes. Science, 294, 581-584.

— E. E. Shuckburgh, W. A. Norton, and N. P. Gillett, 2003: Weather from the stratosphere. Science, 301, 317-318.

Blumen, W., 1979: On short-wave baroclinic instability. J. Atmos. Sci., 36, 1925-1933.

Bretherton, F., 1966: Critical layer instability in baroclinic flows. Quart. J. Roy. Meteor. Soc., 92, 325-334.

Charlton, A. J., A. O'Neill, W. Lahoz, and A. Massacand, 2004: Sensitivity of tropospheric forecasts to stratospheric initial conditions. Quart. J. Roy. Meteor. Soc., 130, 1771-1792.

Christiansen, B., 2003: Temporal growth and vertical propagation of perturbations in the winter atmosphere. Quart. J. Roy. Meteor. Soc., 129, 1589-1605.

Davies, H., and C. Bishop, 1994: Eady edge waves and rapid development. J. Atmos. Sci., 51, 1930-1946.

Hartmann, D. L., 2000: The key role of lower-level meridional shear in baroclinic wave life cycles. J. Atmos. Sci., 57, 389-401.

- , and P. Zuercher, 1998: Response of baroclinic life cycles to barotropic shear. J. Atmos. Sci., 55, 297-313.

_ J. J. Wallace, V. Limpasuvan, D. W. J. Thompson, and J. R. Holton, 2000: Can ozone depletion and greenhouse warming interact to produce rapid climate change? Proc. Natl. Acad. Sci. USA, 97, 1412-1417.

Heifetz, E., J. Methven, B. Hoskins, and C. Bishop, 2004: The counter-propagating Rossby wave perspective on baroclinic instability. Part I: Mathematical basis. Quart. J. Roy. Meteor. Soc., 130, 211-231.

Held, I. M., and M. J. Suarez, 1994: A proposal for the intercomparison of the dynamical cores of atmospheric general circulation models. Bull. Amer. Meteor. Soc., 75, 1825-1830.

Kalnay, E., and Coauthors, 1996: The NCEP/NCAR 40-Year Reanalysis Project. Bull. Amer. Meteor. Soc., 77, 437-471.

Kushner, P. J., and L. M. Polvani, 2004: Stratosphere-troposphere coupling in a relatively simple AGCM: The role of eddies. $J$. Climate, 17, 629-639.

Limpasuvan, V., and D. L. Hartmann, 2000: Wave-maintained annular modes of climate variability. J. Climate, 13, 4414-4429.
—, D. W. J. Thompson, and D. L. Hartmann, 2004: The life cycle of the Northern Hemisphere sudden stratospheric warmings. J. Climate, 17, 2584-2596.

Lindzen, R., 1970: Internal gravity waves in atmospheres with realistic dissipation and temperature. Part I: Mathematical development and propagation of waves into the thermosphere. Geophys. Fluid Dyn., 1, 303-355.

Miller, R. L., G. A. Schmidt, and D. T. Shindell, 2006: Forced annular variations in the 20th century Intergovernmental Panel on Climate Change Fourth Assessment Report models. J. Geophys. Res., 111, D18101, doi:10.1029/2005JD006323.

Müller, J., 1991: Baroclinic instability in a two-layer, vertically semi-infinite domain. Tellus, 43A, 275-284.

Pierrehumbert, R. T., and K. L. Swanson, 1995: Baroclinic instability. Annu. Rev. Fluid Mech., 27, 419-467.

Plumb, R. A., and K. Semeniuk, 2003: Downward migration of extratropical zonal wind anomalies. J. Geophys. Res., 108, 4223, doi:10.1029/2002JD002773.

Polvani, L. M., and P. J. Kushner, 2002: Tropospheric response to stratospheric perturbations in a relatively simple general circulation model. Geophys. Res. Lett., 29, 1114, doi:10.1029/ 2001 GL014284.

— flux near the tropopause. Geophys. Res. Lett., 31, L02115, doi:10.1029/2003GL017965.

Randel, W. J., and I. M. Held, 1991: Phase speed spectra of transient eddy fluxes and critical layer absorption. J. Atmos. Sci., 48, 688-697.

Rivest, C., and C. Davis, 1992: Upper tropospheric synoptic-scale waves. Part I: Maintenance as Eady normal modes. J. Atmos. Sci., 49, 2108-2119.

Rivier, L., R. Loft, and L. M. Polvani, 2002: An efficient spectral dynamical core for distributed memory computers. Mon. Wea. Rev., 130, 1384-1396.

Silverman, B. W., 1986: Density Estimation for Statistics and Data Analysis. Chapman and Hall, 175 pp.

Simmons, A., and B. Hoskins, 1976: Baroclinic instability on the sphere: Normal modes of the primitive and quasi-geostrophic equations. J. Atmos. Sci., 33, 1454-1477.

- , and _ 1980: Barotropic influences on the growth and decay of non-linear baroclinic waves. J. Atmos. Sci., 37, 1679-1684.

Song, Y., and W. A. Robinson, 2004: Dynamical mechanisms for stratospheric influences on the troposphere. J. Atmos. Sci., 61, 1711-1725.

Thompson, D. W. J., and J. M. Wallace, 1998: The Arctic Oscillation signature in the wintertime geopotential height and temperature fields. Geophys. Res. Lett., 25, 1297-1300.

—, J. C. Furtado, and T. G. Shepherd, 2006: On the tropospheric response to anomalous stratospheric wave drag and radiative heating. J. Atmos. Sci., 63, 2616-2629.

Thorncroft, C. D., B. J. Hoskins, and M. E. McIntyre, 1993: Two paradigms of baroclinic wave life-cycle behaviour. Quart. J. Roy. Meteor. Soc., 119, 17-55.

Wittman, M. A. H., L. M. Polvani, and A. J. Charlton, 2005: On the meridional structure of annular modes. J. Climate, 18, 2119-2122.

— - _ R. K. Scott, and A. J. Charlton, 2004: Stratospheric influence on baroclinic lifecycles: Connection to the arctic oscillation. Geophys. Res. Lett., 31, L16113, doi:10.1029/ 2004GL020503.

Yin, J. H., 2005: A consistent poleward shift of the storm tracks in simulations of 21st century climate. Geophys. Res. Lett., 32, L18701, doi:10.1029/2005GL023684. 